\title{
Low oxygen levels contribute to improve photohydrogen production in mixotrophic non-stressed Chlamydomonas cultures
}

\author{
Jose Luis Jurado-Oller ${ }^{1}$, Alexandra Dubini ${ }^{2}$, Aurora Galván¹, Emilio Fernández ${ }^{1}$ and David González-Ballester ${ }^{\text {* }}$
}

\begin{abstract}
Background: Currently, hydrogen fuel is derived mainly from fossil fuels, but there is an increasing interest in clean and sustainable technologies for hydrogen production. In this context, the ability of some photosynthetic microorganisms, particularly cyanobacteria and microalgae, to produce hydrogen is a promising alternative for renewable, clean-energy production. Among a diverse array of photosynthetic microorganisms able to produce hydrogen, the green algae Chlamydomonas reinhardtii is the model organism widely used to study hydrogen production. Despite the well-known fact that acetate-containing medium enhances hydrogen production in this algae, little is known about the precise role of acetate during this process.
\end{abstract}

Results: We have examined several physiological aspects related to acetate assimilation in the context of hydrogen production metabolism. Measurements of oxygen and $\mathrm{CO}_{2}$ levels, acetate uptake, and cell growth were performed under different light conditions, and oxygenic regimes. We show that oxygen and light intensity levels control acetate assimilation and modulate hydrogen production. We also demonstrate that the determination of the contribution of the PSII-dependent hydrogen production pathway in mixotrophic cultures, using the photosynthetic inhibitor DCMU, can lead to dissimilar results when used under various oxygenic regimes. The level of inhibition of DCMU in hydrogen production under low light seems to be linked to the acetate uptake rates. Moreover, we highlight the importance of releasing the hydrogen partial pressure to avoid an inherent inhibitory factor on the hydrogen production.

Conclusion: Low levels of oxygen allow for low acetate uptake rates, and paradoxically, lead to efficient and sustained production of hydrogen. Our data suggest that acetate plays an important role in the hydrogen production process, during non-stressed conditions, other than establishing anaerobiosis, and independent of starch accumulation. Potential metabolic pathways involved in hydrogen production in mixotrophic cultures are discussed. Mixotrophic nutrient-replete cultures under low light are shown to be an alternative for the simultaneous production of hydrogen and biomass.

Keywords: Acetate, Algae, Biofuels, Biomass, Chlamydomonas, DCMU, Hydrogen, Low light, Oxygen

\section{Background}

Chlamydomonas hydrogenase HYDA1 is able to catalyze hydrogen $\left(\mathrm{H}_{2}\right)$ production under anaerobic conditions using protons $\left(\mathrm{H}^{+}\right)$and electrons as substrates; oxygen $\left(\mathrm{O}_{2}\right)$ is a strong inhibitor of both activity and expression

\footnotetext{
*Correspondence: q62gobad@uco.es

1 Departamento de Bioquímica y Biología Molecular, Facultad de Ciencias, Universidad de Córdoba, Campus de Rabanales, Edif. Severo Ochoa,

14071 Córdoba, Spain

Full list of author information is available at the end of the article
}

$[1,2]$. HYDA1 is localized in the chloroplast and its physiological electron donor is the plastidic ferredoxin, PETF or FDX1 (FDX1 throughout) [3-5]. Three pathways for $\mathrm{H}_{2}$ production can be described in Chlamydomonas depending on the electron source and the electron transport pathway to HYDA1. With two of these pathways, the electrons come from photosynthetic electron transport, and they are termed photosystem II (PSII)-dependent and PSII-independent pathways. In the first case, the electrons come from water photolysis at the level of PSII, 
and $\mathrm{O}_{2}$ is generated as a by-product [6-8]. In the PSIIindependent pathway, the electrons come from NADPH $[9,10]$, and reach the plastoquinone $(\mathrm{PQ})$ pool via the $\mathrm{NAD}(\mathrm{P}) \mathrm{H}$ :plastoquinone oxidoreductase, NDA2 [11-14]. Degradation of starch is proposed as the main source for NADPH $[15,16]$. Both electrons routes connect to FDX1 prior to final electron donation to HYDA1. The third pathway mobilizes electrons from the fermentative degradation of endogenous compounds, such as pyruvate derived from starch [16-19]. Fermentative $\mathrm{H}_{2}$ production is linked to the activity of the pyruvate:ferredoxin oxidoreductase (PFR), which can donate electrons to HYDA1 via FDX1 [20].

Chlamydomonas is capable of growing photoautotrophically using light and $\mathrm{CO}_{2}$ as the sole carbon source, or mixotrophically (acetate and light), using acetate as a carbon source. The observation that in some algal species adapted to light and anaerobiosis, $\mathrm{H}_{2}$ photoproduction is enhanced by the presence of acetate in the media was discovered several decades ago [17, 21-24]. These former studies established that under illumination algae can consume the acetate available in the medium and subsequently release $\mathrm{H}_{2}$ and $\mathrm{CO}_{2}$. In the dark, however, acetate has no effect on $\mathrm{H}_{2}$ production, and acetate uptake is not detected. Despite these initial studies, little has been done recently regarding the role of acetate in the production of $\mathrm{H}_{2}$ by algae in nutrient-replete media $[25,26]$.

In this work, we have examined the physiology of photohydrogen production in acetate-containing cultures, and we discuss the importance of controlling acetate assimilation through a fine tune of the $\mathrm{O}_{2}$ and light intensity levels for efficient and sustainable production of $\mathrm{H}_{2}$.

\section{Results}

Acetate-containing media and low light are non-stressful conditions that elicits $\mathrm{H}_{2}$ production

Hermetically sealed vessels containing $100 \mathrm{ml}$ of low cell density $(10 \mu \mathrm{g}$ chl./ml; $\sim 3$ million cells $/ \mathrm{ml})$ Chlamydomonas cells cultured in Tris-Acetate-Phosphate (TAP) were placed under four different light conditions (12, 22, 50 and $100 \mu \mathrm{mol}$ photons $\mathrm{m}^{-2} \mathrm{~s}^{-1}$; hereafter, 12 PAR, 22 PAR, 50 PAR, and 100 PAR) and dark. In this work, we will term 12 and 22 PAR as Low Light (LL), 50 PAR as Moderate Light (ML), and 100 PAR as High Light (HL). The headspaces of the culture vessels (40 ml) were not purged, and therefore atmospheric air was present in all vessels at the beginning of the experiments. $\mathrm{H}_{2}$, $\mathrm{CO}_{2}$ and $\mathrm{O}_{2}$ accumulation in the headspace, chlorophyll content, and acetate consumption were measured daily during 10-day experiments (Fig. 1 and Additional file 1: Fig. S1).

Cultures incubated under LL and ML produced $\mathrm{H}_{2}$ after $24 \mathrm{~h}$, at rates of $6.9,6.0$, and $3.7 \mathrm{ml} \mathrm{L}^{-1}$ day $^{-1}$ for 12, 22, and 50 PAR, respectively (Fig. 1a). After $48 \mathrm{~h}$, little or no $\mathrm{H}_{2}$ production was observed under these light intensities. Interestingly, the $\mathrm{H}_{2}$ accumulation observed under light did not reach a steadystate level, but rather a small decrease was observed after the maximal $\mathrm{H}_{2}$ accumulation was reached. The $\mathrm{H}_{2}$ decrease was most evident under the 12 PAR

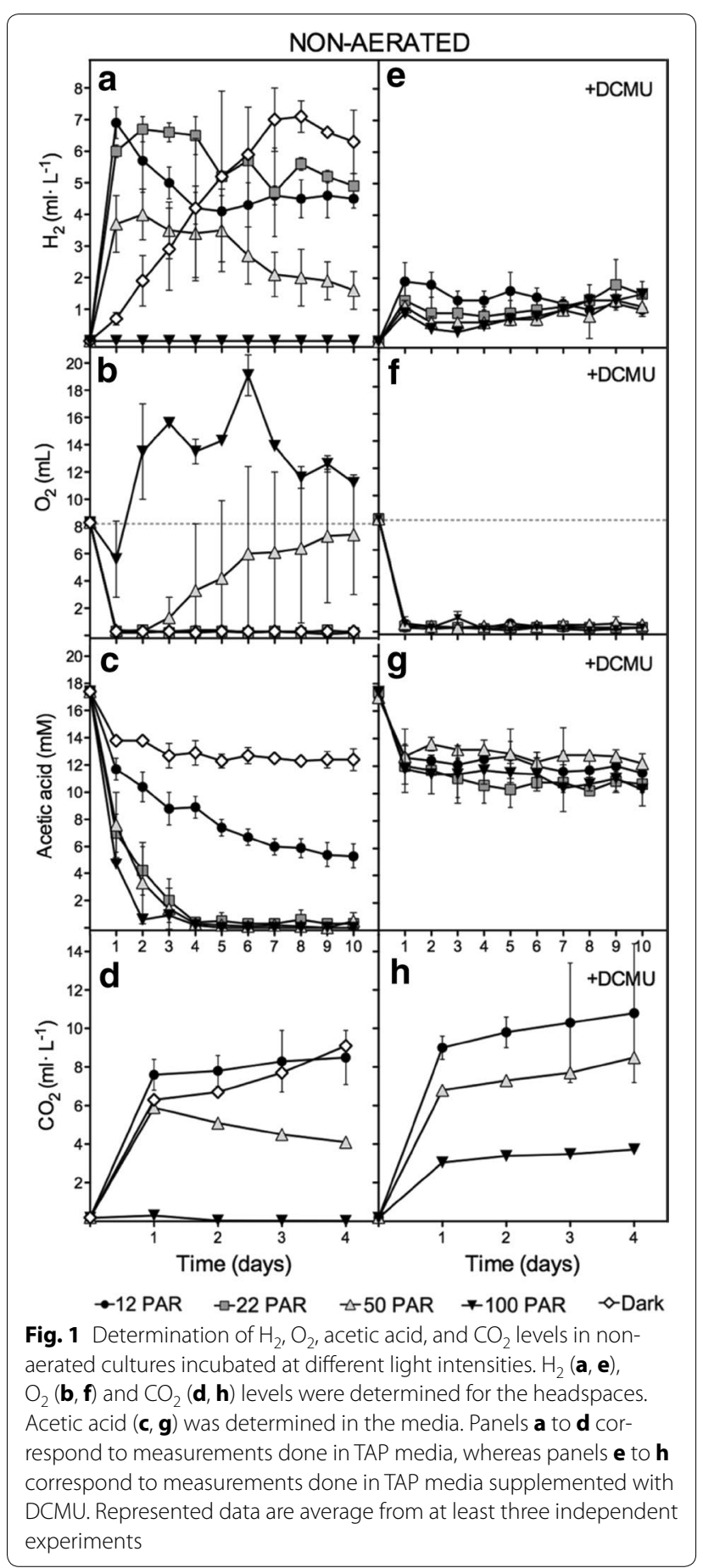


condition. This observation is likely due to $\mathrm{H}_{2}$ uptake activity. Cultures under 100 PAR resulted in no $\mathrm{H}_{2}$ production. Finally, dark cultures had lower initial $\mathrm{H}_{2}$-production rates relative to 12-50 PAR conditions (average less than $1 \mathrm{ml} \mathrm{L}^{-1}$ day $^{-1}$ ), although production was sustained for more than 7 days. Final $\mathrm{H}_{2}$ accumulation in dark cultures, after 8 days, was similar to that obtained under LL after 24-48 $\mathrm{h}$. The atmospheric levels of $\mathrm{O}_{2}$ initially present in the headspaces were totally consumed after $24 \mathrm{~h}$ for all conditions except 100 PAR (Fig. 1b). $\mathrm{O}_{2}$ levels in the headspace remained close to zero for 12, 22 PAR and the dark conditions over the 10 days of the experiment. However, under the 50 PAR conditions, $\mathrm{O}_{2}$ accumulation was observed in the headspace after 3 days. At 100 PAR, $\mathrm{O}_{2}$ evolved above the atmospheric level, indicating that PSII activity overtook respiration rates. Acetate uptake also showed light dependence under our experimental conditions (Fig. 1c). Under 22-100 PAR, all the acetic acid contained in the media was essentially consumed after 2-4 days. However, we observed slower acetate uptake kinetics under 12 PAR and dark conditions. After 10 days, there was still acetic acid in the media $(5.3 \mathrm{mM}$ and $9.5 \mathrm{mM}$ for 12 PAR and dark, respectively). $\mathrm{CO}_{2}$ quickly accumulated after $24 \mathrm{~h}$ in all tested conditions except 100 PAR (Fig. 1d). The media $\mathrm{pH}$ remained stable over the 10 days (7.5-7.8). Finally, the chlorophyll concentration increased significantly under 22-100 PAR, whereas there was very little or no increase at 12 PAR and in the dark, respectively (Additional file 1: Fig. S1A).

These data indicate that acetate-containing cultures grown under $\leq 50$ PAR and moderately low cell density can rapidly consume $\mathrm{O}_{2}$ from the headspaces (for a 100:40 medium: headspace $\mathrm{v} / \mathrm{v}$ ratio), reaching anaerobiosis and producing $\mathrm{H}_{2}$ after $24 \mathrm{~h}$. Maximal $\mathrm{H}_{2}$ production rates were inversely correlated with light intensities. However, dark cultures did not experience a rapid $\mathrm{H}_{2}$-production phase but rather a slow, continued, and sustained level of $\mathrm{H}_{2}$ production. This indicates that light is crucial to induce the rapid $\mathrm{H}_{2}$ production kinetics observed under LL and ML.

\section{Aerations of cultures can double $\mathrm{H}_{2}$ photoproduction}

The same experimental conditions described above were also used to monitoring $\mathrm{H}_{2}$ production in aerated vessels. Aeration was performed by opening the caps of the vessels for $5 \mathrm{~min}$, in a sterile atmosphere, every $24 \mathrm{~h}$. $\mathrm{H}_{2}, \mathrm{CO}_{2}$ and $\mathrm{O}_{2}$ accumulation in the headspace, acetate consumption, and chlorophyll content were measured daily before aeration during 10-day experiments (Fig. 2; Additional file 1: Fig. S1). $\mathrm{H}_{2}$ and $\mathrm{CO}_{2}$ levels are plotted as total production, whereas $\mathrm{O}_{2}$ levels are plotted as

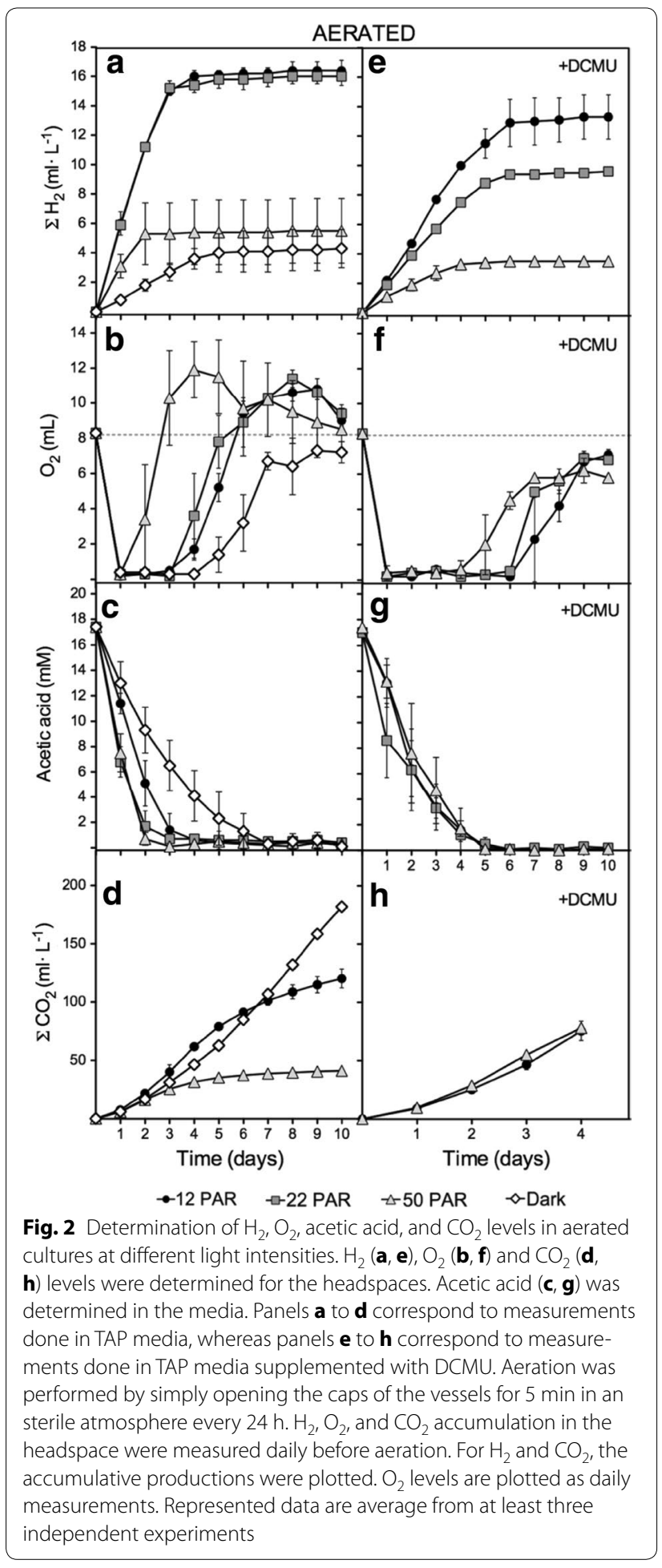

daily measurements. Chlorophyll increased steadily with the intensity of light (Additional file 1: Fig. S1), whereas no changes were observed for $\mathrm{pH}$, which was around 7.6-7.8. 
The $\mathrm{H}_{2}$ production in aerated cultures under LL was 2.4 times higher than in non-aerated cultures under the same light conditions (Figs. 2a vs 1a). For cultures under 50 PAR and dark, the aeration either slightly increased or reduced $\mathrm{H}_{2}$ production, respectively, relative to non-aerated cultures. Under LL, unlike the non-aerated vessels, $\mathrm{H}_{2}$ production was sustained for 3 days. Afterwards, very little $\mathrm{H}_{2}$ production was observed. For LL grown cultures, atmospheric levels of $\mathrm{O}_{2}$ entering in the vessels daily were completely consumed during the first 3 days (Fig. 2b). By the 4th day, increased levels of $\mathrm{O}_{2}$ were detected in the headspace indicating that the $\mathrm{O}_{2}$ consumption rates were progressively decreasing. Similar results were observed for cultures under 50 PAR, although appearance of $\mathrm{O}_{2}$ in the headspaces was detected earlier as the light intensity was increased. For all light intensities, except darkness, headspace $\mathrm{O}_{2}$ accumulation exceeded the initial $\mathrm{O}_{2}$ atmospheric levels, indicating net $\mathrm{O}_{2}$ photoproduction (Fig. 2b). Unlike the non-aerated cultures, all the acetate initially contained in the media was eventually consumed in the aerated cultures under all the conditions tested (Fig. 2c). More specifically, in the light, acetic acid was consumed after 2-3 days. We observed a strong correlation among the $\mathrm{H}_{2}$ production, the level of $\mathrm{O}_{2}$ in the headspace, and the consumption of acetate, indicating that these three processes are closely interconnected. $\mathrm{H}_{2}$ was produced as long as acetate was present in the media. Once acetate was consumed, $\mathrm{O}_{2}$ was accumulated and $\mathrm{H}_{2}$ production ceased. The higher the light intensity, the faster the acetate was consumed, the faster $\mathrm{O}_{2}$ accumulated in the headspace, and the faster $\mathrm{H}_{2}$ production ceased. Notably, increasing the light intensity not only reduced the length of the $\mathrm{H}_{2}$-production phase but also reduced the daily $\mathrm{H}_{2}$-production rates (e.g., $5 \mathrm{ml}$ $\mathrm{L}^{-1}$ day $^{-1}$ for $12 \mathrm{PAR}$ vs $2.6 \mathrm{ml} \mathrm{L}^{-1}$ day $^{-1}$ for 50 PAR). However, in the dark, $\mathrm{H}_{2}$ production dropped drastically reinforcing the idea that light is crucial for the $\mathrm{H}_{2}$ production rates observed in the light. $\mathrm{CO}_{2}$ production was significantly higher for all the conditions tested when comparing to non-aerated cultures (Figs. 2d vs 1d).

\section{Release of $\mathrm{H}_{2}$ partial pressure highly promotes $\mathrm{H}_{2}$ production in mixotrophic LL cultures}

Two possibilities might explain the higher $\mathrm{H}_{2}$ accumulation observed in aerated cultures relative to non-aerated cultures: (1) the increased acetic acid uptake observed in aerated cultures may result in higher $\mathrm{H}_{2}$ production, or (2) release of the $\mathrm{H}_{2}$ partial pressure from the headspace may favor the equilibrium displacement of the reversible reaction catalyzed by hydrogenases toward $\mathrm{H}_{2}$ production and preventing $\mathrm{H}_{2}$ uptake [27]. When the headspaces of 12 PAR grown cultures were $\mathrm{N}_{2}$-purged daily, $\mathrm{H}_{2}$-production rates during the first 3 days were similar to those observed in aerated cultures (Fig. 3a). However, $\mathrm{N}_{2}$-purged cultures kept producing $\mathrm{H}_{2}$ for 10 days, while aerated cultures stopped producing $\mathrm{H}_{2}$ by the 4th day (Fig. 3b). Total $\mathrm{H}_{2}$ production over the 10 days in the purged cultures was 1.4 times higher than in aerated cultures. Interestingly, acetate uptake in purged cultures was severely impaired compared to aerated cultures (Fig. 3b). Purged cultures consumed only around $7 \mathrm{mM}$ of acetic acid but produced more $\mathrm{H}_{2}$ than aerated cultures, which consumed all the acetate initially present in the media (17.4 mM). These data indicate that enhanced $\mathrm{H}_{2}$ production under aeration is mainly due to partial $\mathrm{H}_{2}$ pressure release and not to the increased acetate uptake rates.

To further test which of the two initial hypotheses were true, we added to non-aerated cultures pure $\mathrm{O}_{2}$ daily to the headspaces at atmospheric levels using a syringe (Additional file 2: Fig. S2). Cultures remained hermetically sealed for 9 days without releasing the $\mathrm{H}_{2}$ partial pressure. The results showed high acetate uptake rates, but this did not improve $\mathrm{H}_{2}$ production, which was similar to that obtained for non-aerated cultures. $\mathrm{O}_{2}$ from the headspaces was consumed for 4 days; after 5 days, $\mathrm{O}_{2}$ started to accumulate. This indicates that despite the fact that cultures reached anoxia for 4 days and that acetate was totally consumed, the $\mathrm{H}_{2}$ partial pressure in the headspaces blocked further $\mathrm{H}_{2}$ production.

\section{Acetic acid and $\mathrm{O}_{2}$ supplementation leads to sustained $\mathrm{H}_{2}$ production under LL conditions}

Cultures were subjected to identical experimental conditions described for aerated cultures for 4 days. After 4 days, cultures consumed most of the initial acetate $(17.4 \mathrm{mM})$ contained in the media and did not

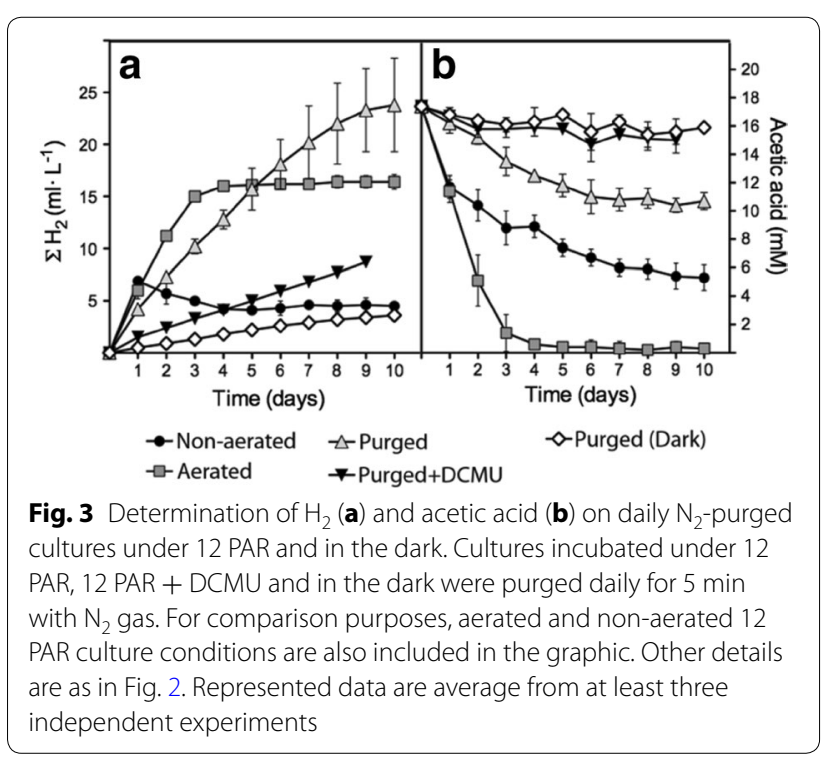


significantly produce $\mathrm{H}_{2}$ afterwards (Fig. 2a). The addition of extra acetic acid (8.7 $\mathrm{mM}$ final concentration) on the 4th day promoted a second boost of $\mathrm{H}_{2}$ production under all light conditions except on cultures kept in the dark (Fig. 4a). After acetic acid supplementation, $\mathrm{H}_{2}$ production was sustained for another one (50 PAR) or two (12 and 22 PAR) days before ceasing again. Addition of a second dose of acetic acid on the 7th day (once $\mathrm{H}_{2}$ production stopped and acetate was consumed again) promoted a 3rd $\mathrm{H}_{2}$-production boost. Supplementation with acetic acid did not significantly change the $\mathrm{pH}$ of the media over the 10 days of the experiment, which remains about 7.5-7.8. In agreement with our previous data, LL grown cultures produced more $\mathrm{H}_{2}$ than 50PAR cultures. At $12 \mathrm{PAR}$, the maximal $\mathrm{H}_{2}$ production rate was $21.2 \mathrm{ml}$ per day, and net total accumulation was 14.9 and 4.0 times higher compared to non-aerated and aerated without supplementation cultures, respectively. Similar to aerated cultures, we observed a good correlation between $\mathrm{H}_{2}$ production, acetate uptake, and $\mathrm{O}_{2}$ consumption in the headspace (Fig. $4 \mathrm{a}-\mathrm{c}$ ). Addition of extra acetic acid to the medium increased $\mathrm{CO}_{2}$ production under all conditions tested, compared to non-supplemented cultures (Figs. 4d vs 2d). Supplementation with acetic acid without performing aeration resulted in cell death (data not shown). This is likely due to the low acetic acid uptake observed in the absence of $\mathrm{O}_{2}$ (see below section), which in turn results in acidification of the media ( $\mathrm{pHs}$ of the dead cultures were of 5.6). Overall, the data showed that $\mathrm{H}_{2}$ production can be sustained in fed-batch type bioreactors under LL with both aeration and acetic acid supplementation.

Acetate uptake depends on $\mathrm{O}_{2}$ availability. The inhibitory effect of DCMU on $\mathrm{H}_{2}$ production under $\mathrm{LL}$ is linked to $\mathrm{O}_{2}$ availability and impairment of acetate uptake

To determine the role of PSII activity in the observed $\mathrm{H}_{2}$ production under our experimental conditions, DCMU was added to the cultures.

The addition of DCMU to the non-aerated cultures incubated under 12-50 PAR produced a substantial reduction of $\mathrm{H}_{2}$ accumulation compared to the cultures containing no DCMU (78.8-69.5\% reduction at $24 \mathrm{~h}$ ) (Fig. 1e vs a). We also observed an impairment in acetate uptake in non-aerated cultures treated with DCMU under all light intensities; the acetate concentration initially dropped but no significant acetate uptake was observed after 24-48 h (Fig. 1g). Interestingly, in aerated cultures, this reduction in $\mathrm{H}_{2}$ production when using DCMU (Fig. 2e) was significantly smaller than the one obtained for non-aerated cultures (e.g., 19.2 and $40.1 \%$ at 12 and 22 PAR, respectively). Moreover, addition of DCMU to aerated cultures caused a much lower

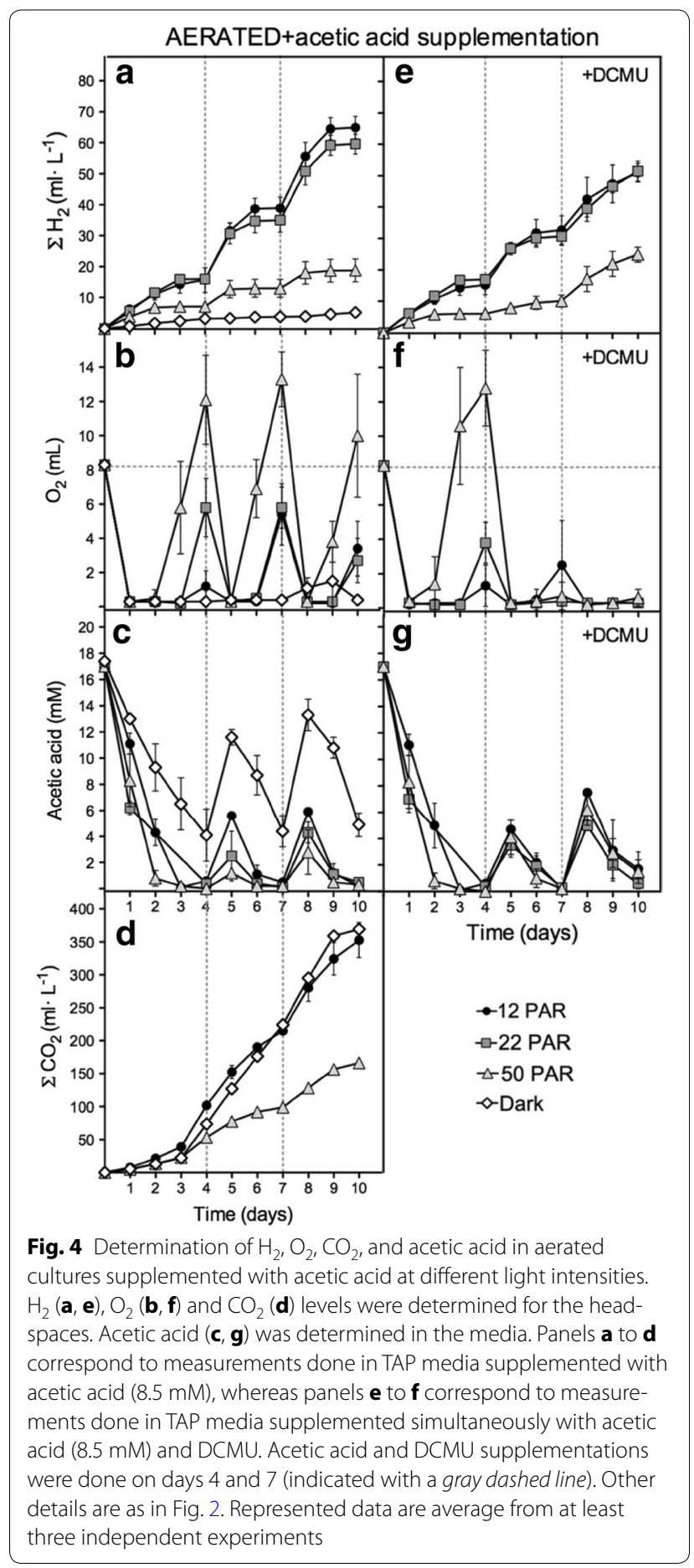

inhibition of the acetate uptake than in non-aerated cultures with DCMU (Figs. $2 \mathrm{~g}$ vs $1 \mathrm{~g}$ ). Indeed, acetate was totally consumed in aerated cultures with DCMU after 4-5 days, while in non-aerated cultures, about $10 \mathrm{mM}$ of acetate remained in the media after 10 days. When daily purged cultures under 12 PAR were supplemented with 
DCMU, both acetate uptake and $\mathrm{H}_{2}$ production dropped severely (Fig. 3). DCMU caused a $64 \%$ inhibition of $\mathrm{H}_{2}$ production, and acetic acid uptake was practically inexistent. The effects of DCMU on $\mathrm{H}_{2}$ production and acetate uptake in purged cultures resemble the observed in non-aerated cultures. Finally, simultaneous addition of both acetic acid and DCMU on the 4th and 7th days in aerated cultures resulted in partial inhibition of $\mathrm{H}_{2}$ production (e.g., $21.5 \%$ reduction for 12 PAR) relative to cultures supplemented with acetic acid but without DCMU (Fig. 4e vs a). However, acetate uptake was not significantly affected in these cultures (Fig. $4 \mathrm{~g}$ vs c). These data are very similar to those obtained with aerated cultures without extra acetic acid supplementation.

Two main conclusions can be obtained from these data. First, we show that acetate uptake depends on the availability of $\mathrm{O}_{2}$. In the presence of DCMU, daily aeration of the cultures can supply the $\mathrm{O}_{2}$ needed to consume all the acetate from the media (Fig. $2 \mathrm{~g}$ ), whereas in non-aerated or purged vessels with DCMU, the lack of $\mathrm{O}_{2}$ inhibits acetate uptake (Fig. 1g). The initial drop in the acetate levels observed in non-aerated cultures containing DCMU after 24-48 $\mathrm{h}$ is likely due to the initial presence of $\mathrm{O}_{2}$ in the non-purged headspace; as $\mathrm{O}_{2}$ level decreased, no more acetate was consumed (Fig. 1g). Moreover, acetate uptake does not depend on the PSII activity per se. However, indirectly, the PSII activity contributes providing $\mathrm{O}_{2}$. This can be observed in non-aerated and aerated cultures without DCMU (Figs. 1c, 2c): daily aeration of the cultures can supply the $\mathrm{O}_{2}$ needed to consume all the acetate from the media, whereas in non-aerated vessels the PSII activity is the only source of $\mathrm{O}_{2}$ that enables acetate uptake. The light dependence of acetate uptake observed in both non-aerated and aerated cultures without DCMU can be explained by the relative activity of the PSII and the availability of the photo-evolved $\mathrm{O}_{2}$. Previous observations describing acetate consumption in the light and termed as photoassimilation of acetate (or acetate photometabolism) [21, 28] must be linked, at least partially, to the photogeneration of $\mathrm{O}_{2}$ but not to the ATP availability.

Second, we demonstrate that DCMU can affect differently $\mathrm{H}_{2}$ production in LL cultures. The addition of DCMU to non-aerated and purged cultures grown under 12 PAR caused very similar effects on inhibition of $\mathrm{H}_{2}$ production (72.4 vs $64 \%$, respectively) (Figs. 1e, 3a) and on acetate uptake (essentially blocked) (Figs. 1g, 3b). The effect of DCMU, however, is clearly different in aerated cultures cultivated under 12 PAR (with or without supplementation with acetic and DCMU) where $\mathrm{H}_{2}$ production was only partially inhibited (nearly $20 \%$ ) (Figs. 2e, $4 \mathrm{e}$ ) and acetate uptake was not severely impaired (Figs. $2 \mathrm{~g}, 4 \mathrm{~g}$ ). Note that the degree of inhibition of the $\mathrm{H}_{2}$ production caused by DCMU correlates with the $\mathrm{O}_{2}$ availability and the rates of acetate uptake. The higher $\mathrm{H}_{2}$ inhibition was obtained when $\mathrm{O}_{2}$ availability and acetate uptake were very low.

Finally, addition of DCMU to non-aerated cultures grown under $\mathrm{HL}$ elicited a small $\mathrm{H}_{2}$ production (Fig. 1e). $\mathrm{H}_{2}$ production was only possible under $\mathrm{HL}$ when DCMU was present in the medium. The presence of this inhibitor was crucial to reach anaerobiosis under HL (Fig. 1f), unlike in the 12-50 PAR grown cultures. Similar results have been found in autotrophic sulfur-depleted cultures [29].

\section{Mobilization of starch reserves are not linked to $\mathrm{H}_{2}$ production in acetate-containing media under $\mathrm{LL}$}

Starch mobilization has been proposed to contribute to $\mathrm{H}_{2}$ production in mixotrophic nutrient-replete cultures via the PSII-independent pathway $[16,17,21]$. It has been suggested that under aerobic conditions acetate would first stimulate starch accumulation, which would be later degraded under anoxic conditions and provide the PQ pool with reductive equivalents. Hence, either the starch reserves present prior the beginning of the experiments or those accumulated during the experiments might potentially contribute to $\mathrm{H}_{2}$ production under LL.

We analyzed the starch accumulation patterns in the LL cultures (Fig. 5). In non-aerated cultures, starch accumulated progressively doubling its initial concentration at the end of the 8-day experiment (Fig. 5a). Similarly, in daily purged cultures under LL, starch initially decreased after $24 \mathrm{~h}$ but then accumulated during 6 days reaching a steady level afterwards (Fig. 5a). In aerated cultures, an initial starch accumulation phase was observed followed by a degradation phase. We observed a good synchronization between the disappearance of acetate from the media (Fig. 2c) and the starch degradation phase

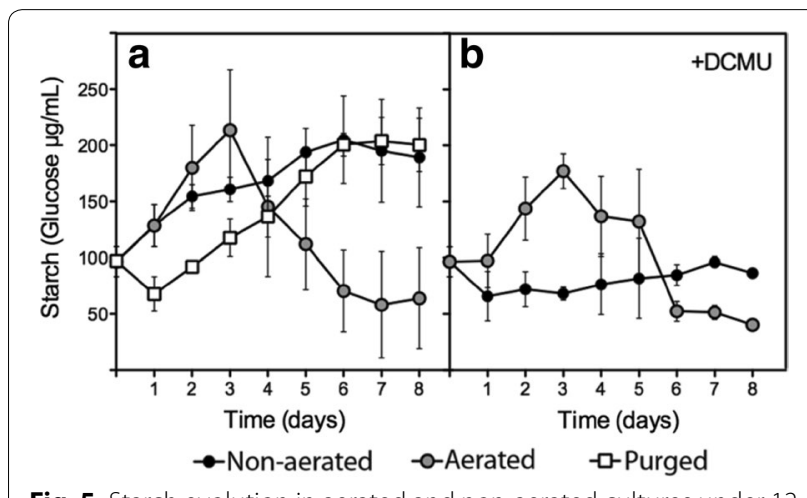

Fig. 5 Starch evolution in aerated and non-aerated cultures under 12 PAR. Starch measurements were performed daily in cultures without $\mathrm{DCMU}(\mathbf{a})$ and with DCMU (b). Represented data are average from at least three independent experiments 
in aerated cultures under LL (Fig. 5a). The addition of DCMU to non-aerated cultures clearly impaired starch accumulation (Fig. 5b), likely reflecting the poor acetate uptake measured in these cultures (Fig. 1g). The addition of DCMU to aerated cultures had only a minor effect on the starch accumulation pattern (Fig. 5b), which is in agreement with the poor inhibition of acetate uptake observed in these cultures (Fig. 2g), and demonstrate that starch accumulation does not require PSII activity but acetate uptake.

Overall, we observed no correlation between starch accumulation/degradation patterns and $\mathrm{H}_{2}$ production because the starch accumulation phases correlated with the maximal $\mathrm{H}_{2}$-production rates in all cases, and there was no further $\mathrm{H}_{2}$ production when starch was degraded (e.g., aerated cultures, see Figs. 5 vs 2 and 2e).

\section{Comparing $\mathrm{H}_{2}$ production in LL mixotrophic nutrient-replete and S-depleted cultures}

$\mathrm{H}_{2}$ production under LL mixotrophic S-depleted conditions was tested using the previously described experimental conditions.

Non-aerated, S-depleted cultures at 12PAR (without purged head space) showed an initial $\mathrm{H}_{2}$-production phase resembling that obtained with $\mathrm{S}$-replete cultures. After 7 days, a second $\mathrm{H}_{2}$-production boost was observed (Supplemental Fig. 3a). While the first $\mathrm{H}_{2}$-production boost might share similar physiological processes with nutrient-replete cultures, the second $\mathrm{H}_{2}$ boost could be more specific to S-deprivation physiology. Interestingly, non-aerated S-depleted cultures presented higher tolerance to the inhibitory effect of the $\mathrm{H}_{2}$ partial pressure than nutrient-replete cultures. Maximal $\mathrm{H}_{2}$ accumulation in non-aerated S-depleted cultures $\left(13.7 \mathrm{ml} \mathrm{L}^{-1}\right)$ was 2 times higher than in non-aerated $\mathrm{S}$-replete cultures $\left(6.9 \mathrm{ml} \mathrm{L}^{-1}\right)$, but similar to the production obtained in aerated S-replete cultures $\left(16.4 \mathrm{ml} \mathrm{L}^{-1}\right)$ (Supplemental Fig. 3b). S-depleted aerated cultures produce no $\mathrm{H}_{2}$ (Additional file 3: Fig. S3B) because cells were unable to consume the $\mathrm{O}_{2}$ that daily entered in the headspaces and anaerobiosis never occurred (data not shown) revealing that $\mathrm{O}_{2}$ consumption rates in S-depleted cultures are lower than in S-replete cultures. S-depleted daily purged cultures showed $\mathrm{H}_{2}$ production rates quite similar to those of S-replete purged cultures (Additional file 3: Fig. S3C).

Overall, the results showed that although $\mathrm{S}$ starvation can improve $\mathrm{H}_{2}$ production in non-aerated cultures, similar levels of $\mathrm{H}_{2}$ can be obtained in S-replete, aerated cultures in a shorter period of time. Moreover, S-depleted, purged cultures did not show any significant advantage over S-replete purged cultures. Hence, under our experimental conditions, removal of $\mathrm{S}$ from the cultures does not contribute a significant advantage over nutrientreplete media under LL.

\section{Discussion}

\section{$\mathrm{H}_{2}$ partial pressure critically inhibits $\mathrm{H}_{2}$ production}

Hydrogenases have a reversible nature and are capable of both biosynthesizing $\mathrm{H}_{2}$ and dissociating it into $\mathrm{H}^{+}$ and electrons. This latter activity is commonly known as $\mathrm{H}_{2}$ uptake. As any chemical reaction, the equilibrium between the biosynthesis and uptake of $\mathrm{H}_{2}$ depends on the concentrations of substrates and products. This would imply that $\mathrm{H}_{2}$ accumulation within bioreactors increases $\mathrm{H}_{2}$ partial pressure in the headspace, while reducing $\mathrm{H}_{2}$ biosynthetic rates to eventually cease any net hydrogenase bioproduction activity.

Inhibition of the Chlamydomonas $\mathrm{H}_{2}$ production by injection of different amounts of pure $\mathrm{H}_{2}$ in the headspaces has previously been observed by others [21, 27]. Moreover, Mignolet et al. [14] demonstrated how $\mathrm{H}_{2}$ elimination from hermetic cultures by flushing with $\mathrm{N}_{2}$ can enhance $\mathrm{H}_{2}$ production. Finally, Kosourov et al. [27] also studied the effect of different volumes of purged headspaces on $\mathrm{H}_{2}$ production in Chlamydomonas cultures under S- and phosphorous-deprivation. Here, we certainly confirm that the release of the $\mathrm{H}_{2}$ accumulated in the headspace greatly enhances $\mathrm{H}_{2}$ production, even when this release is accompanied by $\mathrm{O}_{2}$ supplementation to the cultures (Fig. 2a). Daily release of the $\mathrm{H}_{2}$ accumulated in the headspaces of the bioreactors vessels, through either aeration, or purging with $\mathrm{N}_{2}$, resulted in $\mathrm{H}_{2}$ production 2.4 and 3.4 times higher than in non-aerated cultures, respectively (Fig. 3a). In our experimental designs, release of the $\mathrm{H}_{2}$ partial pressure was executed every $24 \mathrm{~h}$. Since non-aerated cultures did not evolve substantial $\mathrm{H}_{2}$ after $24 \mathrm{~h}$ (Fig. 1a), we conclude that under our nutrient-replete LL conditions, the equilibrium between $\mathrm{H}_{2}$ production and $\mathrm{H}_{2}$ uptake was reached before the 24-h aeration events. In fact, $\mathrm{H}_{2}$ uptake can be observed after $24 \mathrm{~h}$ in LL, non-aerated cultures (Fig. 1a). For dark non-aerated cultures, the equilibrium is reached after 7-8 days (Fig. 1a). However, for both non-aerated LL and dark cultures, the maximal accumulation of $\mathrm{H}_{2}$ in the headspaces reached about $1.9 \%\left(0.76 \mathrm{ml} \mathrm{H}_{2}\right.$ out of $40 \mathrm{ml}$ headspace). Hence, we assume that $1.9 \%$ is near the maximal percentage of $\mathrm{H}_{2}$ that can be accumulated under our specific experimental nutrient-replete conditions.

Interestingly, in S-depleted, non-aerated cultures (Additional file 3: Fig. S3A), the maximal $\mathrm{H}_{2}$ accumulation was higher than in non-aerated S-replete cultures and reached $3.2 \%$ of the headspace. This could indicate that different physiological conditions can probably alter the substrates availability of the hydrogenases $\left(\mathrm{H}^{+}\right.$and electrons) exerting different equilibrium pressures towards $\mathrm{H}_{2}$ formation. 
Overcoming the inhibition of $\mathrm{H}_{2}$ accumulation within bioreactors must be dealt with to optimize $\mathrm{H}_{2}$ production. Utilization of a larger gas to liquid phase ratios or ventilation systems that liberate $\mathrm{H}_{2}$ partial pressure are straightforward ways to overcome the hydrogenase reversibility. However, this may lead the production of highly diluted $\mathrm{H}_{2}$, which may in turn reduce the potential for commercial applications. Utilization of bioreactors covered with materials, such as metallacarboranes, with high reversible capacity for binding $\mathrm{H}_{2}$ at low temperatures [30] might be a way to avoid $\mathrm{H}_{2}$ uptake.

\section{Production of both biomass and $\mathrm{H}_{2}$ is possible in non-stressed Chlamydomonas cultures}

Hydrogenase sensitivity to $\mathrm{O}_{2}$ is probably the main drawback for sustained $\mathrm{H}_{2}$ photoproduction in photosynthetic organisms. Reduction of net $\mathrm{O}_{2}$ levels can be accomplished by reducing PSII activity and maintaining high rates of mitochondrial respiration. At physiological level, nutrient stresses (in the presence of acetate) are widely employed strategies to establish this condition in Chlamydomonas. Sulfur limitation is the most widely employed [6], although phosphorous [31], nitrogen [32] and magnesium [33] deficiencies have been used successfully to attain $\mathrm{H}_{2}$ production in Chlamydomonas. These approaches imply a two-stage process: first biomass is grown under aerobic conditions in nutrient-replete media, and then a $\mathrm{H}_{2}$ production phase can be induced by removing nutrients and $\mathrm{O}_{2}$ from the media. This procedure normally requires a solid-liquid separation step of biomass and culture medium after the growth phase, followed by several washing steps and $\mathrm{O}_{2}$ purging processes $[6,34,35]$. Continuous or semi-continuous regimes of cultivation [36-38] or re-addition of nutrients to the medium [39] are alternatives to the solid-liquid separation steps, which extend the sustainability of $\mathrm{H}_{2}$ production. However, all these protocols normally require high-energy inputs, do not support algae biomass and $\mathrm{H}_{2}$ production simultaneously, are time-consuming and reduce culture viability.

In this work, we show an alternative straightforward methodology for $\mathrm{H}_{2}$ production in nutrient-replete media under LL. This methodology neither requires initial high cell density cultures nor the removal of nutrients from the media. Moreover, under LL and in the presence of acetate, the atmospheric $\mathrm{O}_{2}$ is totally depleted after $24 \mathrm{~h}$, which makes the purging process, required in other strategies, unnecessary. Finally, $\mathrm{H}_{2}$ production can start within $24 \mathrm{~h}$ avoiding the typical lag phase (2-8 days) observed under nutrient-depleted cultures [15, 29, 31-33]. Overall, this strategy opens up new possibilities for production of both $\mathrm{H}_{2}$ and biomass, the latter being important for further downstream biotechnological applications.
Here, we demonstrated that Chlamydomonas, nutrient-replete, mixotrophic, cultures grown under LL $(<50$ PAR) can reach anoxia after $24 \mathrm{~h}$ and produce $\mathrm{H}_{2}$, highlighting the inverse relationship between $\mathrm{H}_{2}$ production rates and light intensities. Similar results have been previously reported by Degrenne et al. [26] who studied the induction of anoxia and $\mathrm{H}_{2}$ production in mixotrophic Chlamydomonas cultures using batch and continuous mode bioreactors under different light regimes. Here, we have extended these previous studies using cultures under different light intensities and oxygenic regimes. We have described in detail how light intensity, $\mathrm{O}_{2}$ availability, acetate uptake, and $\mathrm{H}_{2}$ production are processes closely linked to each other. Here, we have shown that $\mathrm{O}_{2}$ allows acetate uptake and cell growth. Providing $\mathrm{O}_{2}$ to batch mode cultures under LL allowed us to achieve simultaneously $\mathrm{H}_{2}$ production and biomass (Fig. 2a; Additional file 1: Fig. S1). Moreover, in aerated fed-batch mode bioreactors, non-continuous supplementation with acetic acid and $\mathrm{O}_{2}$ greatly enhanced $\mathrm{H}_{2}$ production (Fig. 4a). Likely continuous mode bioreactors, with continuous supply of acetic acid and low levels of $\mathrm{O}_{2}$ (plus other basic nutrients), would allow for sustained $\mathrm{H}_{2}$ and biomass production.

In our study, maximal $\mathrm{H}_{2}$ production was obtained when cultures under 12PAR were not aerated but $\mathrm{N}_{2}-$ purged daily. The purging procedure allowed prolonged $\mathrm{H}_{2}$ production (10 days in purged cultures vs 3 days in aerated cultures) (Fig. 3) with a substantial reduction of the acetate consumption, which is of interest for potential large-scale productions. However, $\mathrm{N}_{2}$-purged cultures did not grow and biomass did not increase, likely because $\mathrm{O}_{2}$ levels were too low. Optimization of these procedures must be achieved to obtain both maximal $\mathrm{H}_{2}$ and biomass production. For example, purging gases that include low concentration of $\mathrm{O}_{2}$ can be finely tuned to allow for high $\mathrm{H}_{2}$ production rates and cell growth.

\section{Roles of acetate and oxygen in the production of $\mathrm{H}_{2}$ under $\mathrm{LL}$ in nutrient-replete cultures}

Most of the studies related to $\mathrm{H}_{2}$ production in Chlamydomonas have been performed with $\mathrm{S}$-depleted acetate-containing media $[6,10,34,40]$. The goal of these studies, however, did not focus on identifying the role of acetate during $\mathrm{H}_{2}$ production. When autotrophic Chlamydomonas cultures are used to produce $\mathrm{H}_{2}$ in either nutrient-replete [41] or S-depleted media [29, 35, 42], $\mathrm{H}_{2}$ production is low compared with media containing acetate, which highlight the importance of acetate in the context of $\mathrm{H}_{2}$ production under these conditions. From studies using nutrient-replete and $\mathrm{S}$-depleted media, it is widely assumed that acetate facilitates $\mathrm{H}_{2}$ production by helping to establish anoxia in the cultures 
through its oxidation via the Tricarboxylic Acid Cycle (TCA) and oxidative phosphorylation $[6,17,21,26,34$, 43]. The addition of acetate to Chlamydomonas cultures decreases photosynthesis efficiency, net $\mathrm{O}_{2}$ evolution, and $\mathrm{CO}_{2}$ fixation, as well as promotes the transition from state I to state II and mitochondrial respiration [44-47]. All these factors help to establish anoxia in sealed cultures. Repression of $\mathrm{CO}_{2}$ fixation may also contribute to $\mathrm{H}_{2}$ production by reducing the competition for electrons, for HYDA1, at the level of FDX1 [10]. In any case, the role of acetate in stimulation of $\mathrm{H}_{2}$ production in the light has not yet been carefully examined.

We have shown that acetate uptake rates are not directly proportional to $\mathrm{H}_{2}$ production rates (e.g., purged cultures vs aerated cultures, Fig. 3a, b). Indeed, the faster the acetate is consumed, the faster $\mathrm{O}_{2}$ accumulates (once acetate is depleted from the media) and the faster $\mathrm{H}_{2}$-production phase is inhibited (e.g., Fig. $2 \mathrm{a}-\mathrm{c}$ ). On the other hand, we have shown that acetate uptake is highly dependent on $\mathrm{O}_{2}$ availability (e.g., comparing aerated and non-aerated cultures with DCMU, Figs. 1g, 2g). Under severe anoxic conditions, such as in non-aerated cultures with DCMU or daily purged cultures with DCMU, there is no acetate uptake and $\mathrm{H}_{2}$ production is very limited (Figs. 1g, e, 3a, b). This suggests that a minimal threshold of acetate uptake is needed to support $\mathrm{H}_{2}$ production and that the role of acetate must rely on something else than just the establishment of anoxic condition. Moreover, paradoxically, $\mathrm{O}_{2}$ is a key substrate that under limiting concentrations allows acetate uptake and thereby $\mathrm{H}_{2}$ production. Acetate uptake does not directly depend on the PSII activity per se (Fig. 2g); however, PSII activity is crucial to provide $\mathrm{O}_{2}$ to hermetically sealed cultures and allow acetate uptake (e.g., Fig. 1c). Overall, our results suggest that an important aspect to be considered when producing $\mathrm{H}_{2}$ in mixotrophic Chlamydomonas cultures is that optimal $\mathrm{H}_{2}$ production does not rely on reaching very high respiration rates or establishing severe anoxic conditions but rather on sustaining relatively low acetate uptake rates under hypoxic conditions. LL is an optimal physiological condition where $\mathrm{O}_{2}$ production is high enough to allow low acetate uptake rates but low enough to prevent hydrogenase inhibition.

The $\mathrm{H}_{2}$ production observed in acetate-containing media is clearly enhanced in the light, although, under our experimental conditions, dark fermentation may contribute to about $10-20 \%$ of the total $\mathrm{H}_{2}$ production detected at 12PAR. The stimulatory effect of light on $\mathrm{H}_{2}$ production necessarily requires an active photosynthetic electron flow that can feed electrons to HYDA1 via either the PSII-dependent or PSII-independent pathway. The inhibitory effect of DCMU on algal $\mathrm{H}_{2}$ photoproduction has frequently been used to differentiate between
PSII-dependent and -independent pathways [7, 10, 48]. Previously, it was observed that the effect of DCMU on cells grown in nutrient-replete media was unclear. Some studies reveal no impact of DCMU on $\mathrm{H}_{2}$ photoproduction [49]. However, others observed substantial inhibition of both $\mathrm{H}_{2}$ production (90\%) and acetate uptake (66\%) [21, 24]. Here, we demonstrate that the addition of DCMU to nutrient-replete, acetate-containing cultures can have very different effects on both $\mathrm{H}_{2}$ production (e.g., at 12 PAR, inhibition of $72.4 \%$ in non-aerated cultures vs $19.2 \%$ in aerated cultures) and acetate uptake (e.g., at any light intensity, severe inhibition in non-aerated cultures vs slight inhibition in aerated cultures). The level of inhibition of $\mathrm{H}_{2}$ production by DCMU is subjected to the specific culture conditions, more specifically to $\mathrm{O}_{2}$ availability and acetate uptake. This may be problematic for determining the contribution of the PSII-dependent pathway on cells grown in media containing acetate. Experimental conditions (e.g. $\mathrm{O}_{2}$ availability, pre-culture conditions, purging processes, etc.) may affect the effect of DCMU addition on $\mathrm{H}_{2}$ production and in turn overestimate the contribution of the PSII-dependent pathway. This may partly explain some discrepancies found in the literature concerning the relative importance of this pathway. Nonetheless, we conclude that $\mathrm{H}_{2}$ production in LL mixotrophic cultures is mainly via PSII-independent pathway, since up to $80 \%$ of the maximal $\mathrm{H}_{2}$ production can be obtained when PSII is inhibited (Figs. 2e, 4e). It has been proposed that acetate may enhance the PSII-independent $\mathrm{H}_{2}$ production pathway under nutrient-replete media by favoring the accumulation of starch during oxygenic conditions, which is degraded later during anaerobiosis [17, 24, 49, 50]. However, our data do not seem to support this possibility since we observe no correlation between starch accumulation/degradation patterns and $\mathrm{H}_{2}$ production under our experimental conditions (Figs. 3a, 5). Our data are in agreement with previous observations of starch-deficient mutants that can produce $\mathrm{H}_{2}$ under both $\mathrm{S}$-replete and S-depleted conditions [10]. Alternatively, starch degradation may not be the source of reductive equivalents but rather acetate assimilation may provide the chloroplast with reductive equivalents for PSII-independent $\mathrm{H}_{2}$ photoproduction. The localization of two enzymes involved in acetate assimilation, succinate dehydrogenase (SDH) and malate dehydrogenase (MDH), in the chloroplast of Chlamydomonas, may provide this organelle with the reductive equivalents (FADH ${ }_{2}$ and $\mathrm{NADPH}$, respectively) needed to feed the PQ pool [51, 52] (Fig. 6). Additionally, the oxaloacetate produced in the chloroplast from MDH can also potentially contribute to $\mathrm{H}_{2}$ production. Recently, it was shown that the pyruvate:ferredoxin oxidoreductase (PFR) enzyme of Chlamydomonas possesses 


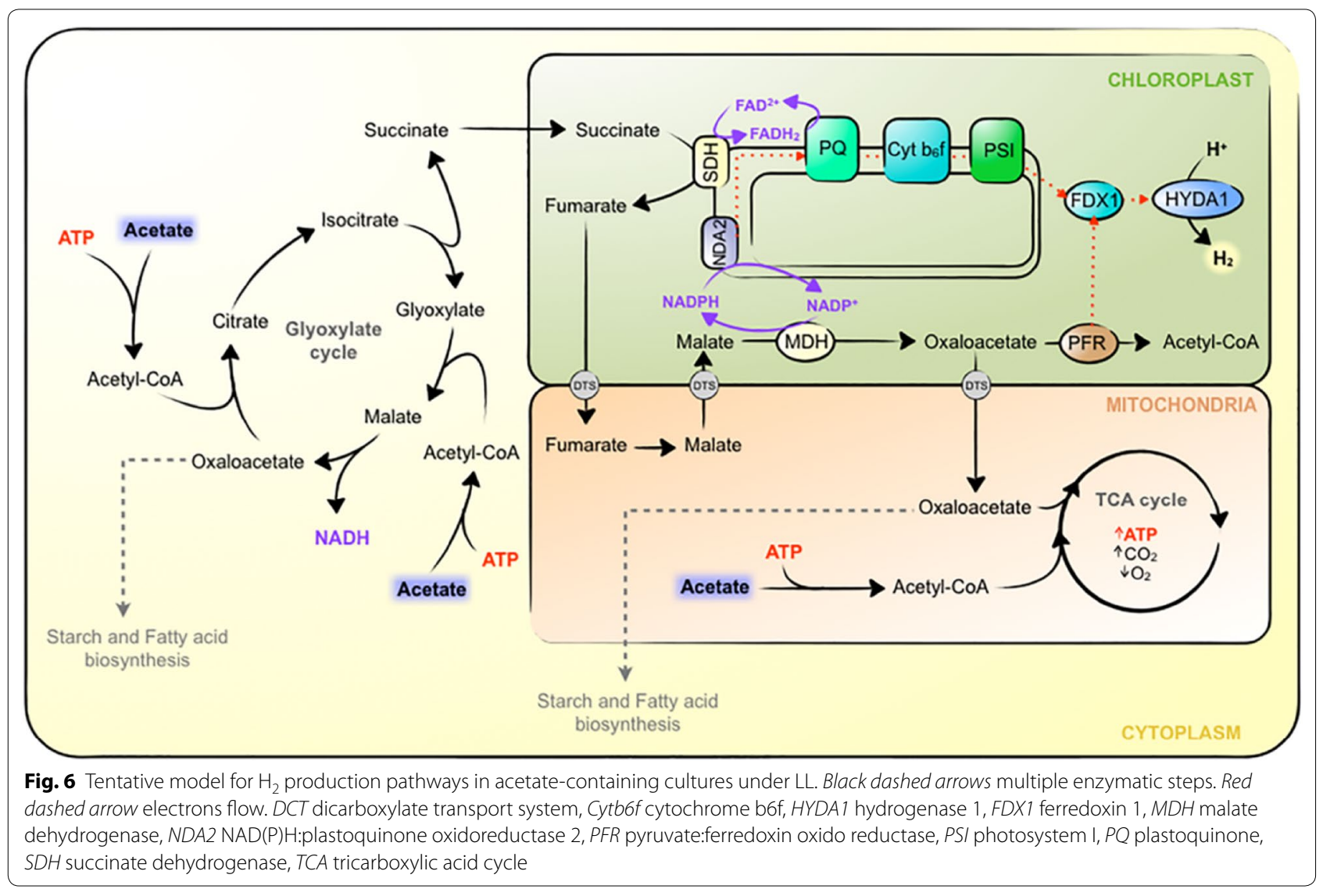

an important affinity for oxaloacetate [53], which opens up the possibility that acetate, via the glyoxylate pathway, might also be coupled to fermentative $\mathrm{H}_{2}$ production (Fig. 6).

In any case, under our experimental conditions, the potential contribution of these pathways (either PSIIindependent via SDH/MDH or fermentative via PFR) needs to be necessarily linked to light-dependent reactions since dark $\mathrm{H}_{2}$ production is clearly lower than in the light. For the SDH/MDH pathway, it is easy to explain the light dependence since electron flow from the PQ pool to HYDA1 would require an active PSI. At the same time, an active PSI would promote PQ re-oxidation and would allow the turnover of $\mathrm{FAD}^{+}$and $\mathrm{NADP}^{+}$needed to maintain SDH and MDH activities, respectively, which in turn would provide oxaloacetate to the chloroplast, leading to high PFR rates under anoxia. Still, the relative importance, if any, of these potential pathways for $\mathrm{H}_{2}$ production in nutrient-replete acetate-containing cultures, remains to be determined appropriately.

Enzyme localization and proteomics studies have helped in identifying the acetate assimilation and dissimilation pathways, which require the participation of enzymes localized in the cytosol, the mitochondria, and the chloroplast [51, 52, 54-56]. Based on these studies and on the physiological data we have provided in this study, we propose a tentative model that aims at explaining the physiology and metabolic pathways involved in $\mathrm{H}_{2}$ production in nutrient-replete, acetate-containing medium (Fig. 6). Acetate can be simultaneously routed to different pathways. Acetate can be dissimilated in the TCA cycle or assimilated by the glyoxylate cycle. The acetate entering the TCA cycle would provide energy to the cell and would contribute in maintaining low $\mathrm{O}_{2}$ levels. The glyoxylate cycle would provide succinate to the chloroplast for carbon skeletons. Chloroplastic SDH and $\mathrm{MDH}$ would participate in the conversion of chloroplast succinate into oxaloacetate and would provide reductive equivalents to the PQ pool. Chloroplastic oxaloacetate would either be redirected towards different biosynthetic pathways (e.g., lipids or starch biosynthesis) or used by chloroplast PFR if anoxic/hypoxic conditions are established. All these pathways occur simultaneously and their relative activities can be affected by $\mathrm{O}_{2}$ availability. Light intensity greatly affects $\mathrm{O}_{2}$ availability through PSII activity, and this $\mathrm{O}_{2}$ could be used by the TCA cycle to dissimilate acetate. If the light intensity is not too high, then the TCA cycle would be able to maintain the cells under 
hypoxic conditions. However, even though hypoxic conditions are established, $\mathrm{O}_{2}$ photoproduction rates would regulate the TCA cycle activity, acetate uptake rate, and cell growth. The higher these parameters, the lower would be the $\mathrm{H}_{2}$ production. Under LL, however, $\mathrm{O}_{2}$ availability is very low and so would be the TCA activity, acetate uptake rates, and ATP generation. Under this scenario, the relative importance of the glyoxylate cycle is enhanced, as well as chloroplastic SDH and MDH activities, which could provide reductive equivalents to the PQ pool. Also, due to low ATP levels, the use of oxaloacetate for biosynthetic pathways and cell growth is impaired and PFR activity is favored. All these processes would favor the $\mathrm{H}_{2}$ production under LL in acetate-containing medium. Under severe anoxic conditions, no acetate would be taken up, and $\mathrm{H}_{2}$ production would be low. Finally, in the dark, and independent of $\mathrm{O}_{2}$ availability, PSII-independent $\mathrm{H}_{2}$ production is not active, and the turnover of $\mathrm{FADH}_{2}$ and NADPH would limit oxaloacetate availability and its use by PFR.

\section{Conclusion}

$\mathrm{H}_{2}$ production by Chlamydomonas cultures is still far for large-scale implementations due to the low yields obtained so far. Among other limitations, the $\mathrm{H}_{2}$ partial pressure is an important limiting factor, as shown in this work. Moreover, most of the studies about $\mathrm{H}_{2}$ production on Chlamydomonas rely in the use of nutrient starved cultures, which reduces the viability of large-scale productions. The approach presented here, although also has very low $\mathrm{H}_{2}$ production yields, opens up a new possibility to the straightforward attainment of both $\mathrm{H}_{2}$ and biomass in non-stressed cultures. This could have a great biotechnological interest if current $\mathrm{H}_{2}$ production yields are sufficiently improved. Another factor limiting $\mathrm{H}_{2}$ production in algae is the $\mathrm{O}_{2}$ sensitivity of the hydrogenases. However, an intriguing conclusion of this work is that low levels of $\mathrm{O}_{2}$ can actually benefit $\mathrm{H}_{2}$ production by the means of facilitating acetate uptake in mixotrophic cultures. However, the role of acetate during the $\mathrm{H}_{2}$ photoproduction in Chlamydomonas cultures remains uncertain. We have shown that $\mathrm{H}_{2}$ production in mixotrophic cultures is mostly associated with the PSII-independent pathway (80\%), indicating that a source of reductants is needed to feed the PQ pool. However, although acetate assimilation can favor starch accumulation, we clearly demonstrate that mobilization of the starch reserves does not provide with reductants for $\mathrm{H}_{2}$ production under our experimental conditions. We discuss potential metabolic pathways that may be involved in $\mathrm{H}_{2}$ production and linked to the dissimilation/assimilation of acetate, but certainly, more studies are needed to unravel the precise mechanisms that trigger $\mathrm{H}_{2}$ production in the presence of acetate in Chlamydomonas cultures. Understanding such mechanisms could help us improving $\mathrm{H}_{2}$ production efficiency through new physiological manipulations and genetic engineering.

\section{Methods}

\section{Alga strain and growth conditions}

Chlamydomonas (Chlamydomonas reinhardtii) strain $704\left(\mathrm{cw} 15 \arg ^{+}\right.$Nia1:Ars $\mathrm{mt}^{+}$) [57] was used in all the experiments. Cells were grown photomixotrophically under continuous light at $23{ }^{\circ} \mathrm{C}$ in liquid TAP medium. The initial acetic acid concentration was of $17.4 \mathrm{mM}$ [58]. Algal cultures were grown to mid-log phase $\left(10-12 \times 10^{6}\right.$ cells $\mathrm{mL}^{-1}$ ), harvested by centrifugation (3000 $g$ for $2 \mathrm{~min}$ ) and resuspended in fresh TAP to a final concentration of $10 \mu \mathrm{g} \mathrm{Chl} \mathrm{mL}{ }^{-1}\left(\sim 3 \times 10^{6}\right.$ cells $\left.\mathrm{mL}^{-1}\right)$. For experiments employing either TAP without S (TAP-S) or acetate free media (Minimal Media, MM) [58], cells were washed two times in the corresponding media before setting up the inductions. Cells $(100 \mathrm{ml})$ were placed in a hermetically sealed vessel $(140 \mathrm{ml})$ using screw caps equipped with silicon septa (GL45 PP 2 ports, GL14, Duran Group). The final headspace volume was $40 \mathrm{~mL}$. The sealed vessels were placed in a growth chamber equipped with LED panels (AlgaeTron AG 230, Photon System Instruments) at $23{ }^{\circ} \mathrm{C}$ with continuous agitation $(210 \mathrm{rpm})$. The following light intensities were assessed: $12,22,50$, and $100 \mu \mathrm{E}$ $\mathrm{m}^{-2} \mathrm{~s}^{-1}$. For dark inductions, the vessels were covered with aluminum foil. Where indicated, the following reagents were added to the cultures: the PSII inhibitor, DCMU (25 $\mu \mathrm{M}$ of 3-(3,4-dicholorophenyl)-1,1-dimethylurea in ethanol) (Sigma-Aldrich); $8.5 \mathrm{mM}$ glacial acetic acid (Panreac); and $8.5 \mathrm{mM}$ potassium acetate. Daily samples $(1 \mathrm{ml})$ of the cultures were collected using a syringe; and they were used for starch, chlorophyll, acetate, and $\mathrm{pH}$ determinations.

\section{Analysis of the gases in the head spaces of the cultures}

Samples $(250 \mu \mathrm{L})$ from the headspaces of the cultures were collected using a $1 \mathrm{~mL}$ Hamilton's SampleLock ${ }^{\mathrm{TM}}$ syringe (\# 81356) and manually injected into a Gas Chromatographer (GC) (Agilent 7820A, Agilent Technologies). $\mathrm{H}_{2}, \mathrm{O}_{2}$ and $\mathrm{CO}_{2}$ were detected using a Thermal Conductivity Detector (TCD), and argon was used as the carrier gas. $\mathrm{H}_{2}, \mathrm{O}_{2}$, and $\mathrm{CO}_{2}$ were separated at $40{ }^{\circ} \mathrm{C}$ using a two-capillary-column system (HP-molesieve $30 \mathrm{~m} \times 0.53 \mathrm{~mm}$ and HP-PLOT/Q $30 \mathrm{~m} \times 0.53 \mathrm{~mm}$, Ref. 19095P-MS6/19095P-QO4, Agilent Technologies) coupled with a pneumatic valve. Alternatively, $\mathrm{O}_{2}$ and $\mathrm{H}_{2}$ were separated using a packed column (60/80 Molecular Sieve 5A, Ref. 13133-U, Supelco) at $70{ }^{\circ} \mathrm{C}$. 


\section{Chlorophyll, acetate, and starch measurements}

Culture samples $(1 \mathrm{~mL})$ were taken directly from culture vessels at the indicated times, centrifuged for $2 \mathrm{~min}$ at $13,000 \mathrm{rpm}$, and the pellets were resuspended in methanol for chlorophyll $(\mathrm{a}+\mathrm{b})$ extraction [58]. Supernatants were stored at $-20{ }^{\circ} \mathrm{C}$ separately for acetate determinations. The concentrations of chlorophylls a and $b$, extracted from cells in methanol, were estimated spectrophotometrically (DU 800, Beckman Coulter).

Acetic acid analysis was performed by HPLC (Agilent series 1200, Agilent Technologies) using an ion-exchange column (Agilent Hi-Plex H, $300 \times 7.7$ mm, $6 \mu \mathrm{m}$ I.D.) and isocratic elution with $5 \mathrm{mM} \mathrm{H}_{2} \mathrm{SO}_{4}$ at $55{ }^{\circ} \mathrm{C}$. Samples were thawed, centrifuged, and filtered prior to HPLC analysis. Twenty $\mu \mathrm{l}$ of cell culture supernatant was injected onto the HPLC system at a flow rate of $0.6 \mathrm{ml} /$ min. Retention peaks were observed using a UV (205 nm) detector and recorded using Agilent ChemStation software. Quantifications were performed by comparisons with known amounts of standard

Starch extraction was performed using a version of the method of Klein and Betz [17]. One milliliter of culture was sampled, centrifuged at 13,000 rpm for $1 \mathrm{~min}$, resuspended in $1 \mathrm{~mL}$ of methanol for chlorophyll extraction, and centrifuged again. The pellets were rinsed with $400 \mu \mathrm{L}$ of distilled water and heated for $15 \mathrm{~min}$ at $120{ }^{\circ} \mathrm{C}$ for starch solubilization. Two hundred microliter of a mixture of $\alpha$-amylase (A-9857, SIGMA) and amyloglucosidase (10115, SIGMA) containing $2.25 \mathrm{U} / \mathrm{mL}$ each, prepared in Na-acetate buffer (100 mM, pH 4.5), were added to the samples. The mixture reaction was incubated for $2 \mathrm{~h}$ at $55^{\circ} \mathrm{C}$. Finally, free glucose was measured using a BioSystems kit for glucose determination (11538, BioSystems)

\section{Additional files}

Additional file: Figure S1. Chlorophyll evolution. A, non-aerated cultures; $B$, aerated cultures; $C$, aerated cultures supplemented with acetic acid; D, non-aerated cultures + DCMU; E, aerated cultures + DCMU; F, aerated cultures supplemented with acetic acid + DCMU. For conditions $\mathrm{C}$ and $\mathrm{F}$ acetic acid $(8.5 \mathrm{mM})$ and DCMU supplementations were done on days 4 and 7 . Represented data are average from at least three independent experiments.

Additional file 2: Figure S2. Effect of daily $\mathrm{O}_{2}$ addition to hermetic cultures. $\mathrm{H}_{2}(\mathrm{~A}), \mathrm{O}_{2}$ (B) levels where determined for the headspaces. Acetic acid (C) was determined in the media. Daily addition of $8.4 \mathrm{ml}$ of pure $\mathrm{O}_{2}$ to the $40 \mathrm{ml}$ headspaces ( $21 \%$ of $\mathrm{O}_{2}$ into the headspace) was obtained through syringe injection across the septum vessels. Cultures were incubated under 12 PAR. For comparison purposes non-aerated 12 PAR cultures condition is also include in the graphic. Represented data are average from at least three independent experiments.

Additional file3: Figure S3. Determination of $\mathrm{H}_{2}$ in $\mathrm{S}$-depleted cultures under $12 \mathrm{PAR} . \mathrm{H}_{2}$ measurements were done in non-aerated (A), aerated (B) and daily purged (C) cultures. The corresponding controls for S-replete conditions are also represented. For aerated and purged cultures $\mathrm{H}_{2}$ accumulative productions are plotted. Represented data are average from at least three independent experiments.

\begin{abstract}
Abbreviations
HL: high light (100 PAR); HYDA1: hydrogenase 1; FDX1: ferredoxin 1; LL: low light (12-22 PAR); MDH: Malate Dehydrogenase; ML: moderate light (50 PAR); NDA2: NAD(P)H:plastoquinone oxidoreductase 2; PAR: photosynthetically active radiation (in $\mu \mathrm{mol}$ photons $\mathrm{m}^{-2} \mathrm{~s}^{-1}$ ); PFR: pyruvate:ferredoxin oxido Reductase; PQ: plastoquinone; SDH: succinate dehydrogenase; TAP: trisacetate-Phosphate medium; TCA: tricarboxylic Acid cycle.
\end{abstract}

\section{Authors' contributions}

$J\llcorner J-O$ and DG-B performed and designed all the experiments. Jப-O helped in the writing of the manuscript and made the final versions of the figures. $A D$ collected some samples, helped in designing of the experiments, in the analysis and interpretation of the data, and in the writing of the manuscript. EF and AG coordinated the study and helped in the writing of the manuscript. DG-B performed the experiments, analyzed the results, interpreted the data and wrote the manuscript. All authors read and approved the final manuscript.

\section{Author details}

${ }^{1}$ Departamento de Bioquímica y Biología Molecular, Facultad de Ciencias, Universidad de Córdoba, Campus de Rabanales, Edif. Severo Ochoa, 14071 Córdoba, Spain. ${ }^{2}$ Biosciences Center, National Renewable Energy Laboratory (NREL), 15013 Denver West Parkway, Golden, CO 80401, USA.

\section{Acknowledgements}

Authors thanks Maria Ghirardi and Michael Seibert (National Renewable Energy Laboratory, CO, USA) for their critical reading of the manuscript; and Maribel Macias and Rocio Onieva (Universidad de Cordoba) for their technical support. This work was funded by the MINECO (Ministerio de Economia y Competitividad, Spain, Grant no. BFU2011-29338 [granted to EF and G]), supported by the European "Fondo Europeo de Desarrollo Regional (FEDER)" program, the Plan E program (CONV 188/09 [granted to EF]), the Ramon y Cajal program (RYC-2011-07671 [granted to DG-B]), the Junta de Andalucía grants (P08-CVI-04157 and BIO-502 [granted to AG and EF]), the Plan Propio de la Universidad de Cordoba (granted to EF and AG), and the U. S. Department of Energy, Office of Biological and Environmental Research (BER) (granted to AD).

\section{Compliance with ethical guidelines}

\section{Competing interests}

The authors declare that they have no competing interests.

Received: 30 April 2015 Accepted: 10 September 2015

Published online: 17 September 2015

\section{References}

1. Happe T, Kaminski A. Differential regulation of the Fe-hydrogenase during anaerobic adaptation in the green alga Chlamydomonas reinhardtii. Eur J Biochem. 2002;269:1022-32.

2. Ghirardi ML, Togasaki RK, Seibert M. Oxygen sensitivity of algal $\mathrm{H}_{2}$-production. Appl Biochem Biotechnol. 1997;63-65:141-51.

3. Peden EA, Boehm M, Mulder DW, Davis R, Old WM, King PW, et al. Identification of global ferredoxin interaction networks in Chlamydomonas reinhardtii. J Biol Chem. 2013;288:35192-209.

4. Terauchi AM, Lu SF, Zaffagnini M, Tappa S, Hirasawa M, Tripathy JN, et al. Pattern of expression and substrate specificity of chloroplast ferredoxins from Chlamydomonas reinhardtii. J Biol Chem. 2009;284:25867-78.

5. Tagawa K, Tsujimoto HY, Arnon DI. Role of chloroplast ferredoxin in the energy conversion process of photosynthesis. Proc Natl Acad Sci USA. 1963:49:567-72

6. Melis A, Zhang L, Forestier M, Ghirardi ML, Seibert M. Sustained photobiological hydrogen gas production upon reversible inactivation of oxygen evolution in the green alga Chlamydomonas reinhardtii. Plant Physiol. 2000;122:127-36.

7. Kosourov S, Seibert M, Ghirardi ML. Effects of extracellular pH on the metabolic pathways in sulfur-deprived, $\mathrm{H}_{2}$-producing Chlamydomonas reinhardtii cultures. Plant Cell Physiol. 2003;44:146-55. 
8. Greenbaum E, Lee JW, Tevault CV, Blankenship SL, Mets LJ. CO2 fixation and photoevolution of $\mathrm{H} 2$ and $\mathrm{O} 2$ in a mutant of Chlamydomonas lacking photosystem I. Nature. 1995;376:438-41.

9. Mus F, Cournac L, Cardettini V, Caruana A, Peltier G. Inhibitor studies on non-photochemical plastoquinone reduction and $\mathrm{H}(2)$ photoproduction in Chlamydomonas reinhardtii. Biochim Biophys Acta. 2005;1708:322-32.

10. Hemschemeier A, Fouchard S, Cournac L, Peltier G, Happe T. Hydrogen production by Chlamydomonas reinhardtii: an elaborate interplay of electron sources and sinks. Planta. 2008;227:397-407.

11. Baltz A, Kieu-Van D, Beyly A, Auroy P, Richaud P, Cournac L, et al. Plastidia expression of type II NAD(P)H dehydrogenase increases the reducing state of plastoquinones and hydrogen photoproduction rate by the indirect pathway in Chlamydomonas reinhardtii. Plant Physiol. 2014;165:1344-52.

12. Desplats C, Mus F, Cuine S, Billon E, Cournac L, Peltier G. Characterization of $\mathrm{Nda} 2$, a plastoquinone-reducing type II NAD(P)H dehydrogenase in Chlamydomonas chloroplasts. J Biol Chem. 2009;284:4148-57.

13. Jans F, Mignolet E, Houyoux P-A, Cardol P, Ghysels B, Cuine S, et al. A type II NAD(P) $H$ dehydrogenase mediates light-independent plastoquinone reduction in the chloroplast of Chlamydomonas. Proc Nat Acad Sci USA. 2008;105:20546-51.

14. Mignolet E, Lecler R, Ghysels B, Remacle C, Franck F. Function of the chloroplastic $\mathrm{NAD}(\mathrm{P}) \mathrm{H}$ dehydrogenase $\mathrm{Nda} 2$ for $\mathrm{H}_{2}$ photoproduction in sulphur-deprived Chlamydomonas reinhardtii. J Biotech. 2012;162:81-8.

15. Chochois V, Dauvillée D, Beyly A, Tolleter D, Cuiné S, Timpano H, et al. Hydrogen production in Chlamydomonas: photosystem II-dependent and -independent pathways differ in their requirement for starch metabolism. Plant Physiol. 2009;151:631-40.

16. Gfeller RP, Gibbs M. Fermentative Metabolism of Chlamydomonas reinhardtii: II. Role of Plastoquinone. Plant Physiol. 1985;77:509-11.

17. Klein U, Betz A. Fermentative Metabolism of Hydrogen-evolving Chlamydomonas moewusii. Plant Physiol. 1978;61:953-6.

18. Hemschemeier A, Happe T. The exceptional photofermentative hydrogen metabolism of the green alga Chlamydomonas reinhardtii. Biochem Soc Trans. 2005;33:39-41.

19. Mus F, Dubini A, Seibert M, Posewitz MC, Grossman AR. Anaerobic acclimation in Chlamydomonas reinhardtii: anoxic gene expression, hydrogenase induction, and metabolic pathways. J Biol Chem. 2007;282:25475-86

20. van Lis R, Baffert C, Couté Y, Nitschke W, Atteia A. Chlamydomonas reinhardtii chloroplasts contain a homodimeric pyruvate:ferredoxin oxidoreductase that functions with FDX1. Plant Physiol. 2013;161:57-71.

21. Gibbs M, Gfeller RP, Chen C. Fermentative metabolism of Chlamydomonas reinhardii: III. Photoassimilation of acetate. Plant Physiol. 1986;82:160-6.

22. Healey FP. Mechanism of hydrogen evolution by Chlamydomonas moewusii. Plant Physiol. 1970;45:153-9.

23. Jones LWMJ. A common link between photosynthesis and respiration in a blue green alga Nature. 1963;199:670-2.

24. Bamberger ES, King D, Erbes DL, Gibbs M. H(2) and CO(2) Evolution by anaerobically adapted Chlamydomonas reinhardtii F-60. Plant Physiol. 1982;69:1268-73.

25. Wang H, Fan X, Zhang Y, Yang D, Guo R. Sustained photo-hydrogen production by Chlorella pyrenoidosa without sulfur depletion. Biotechnol Lett. 2011;33:1345-50.

26. Degrenne B, Pruvost J, Christophe G, Cornet JF, Cogne G, Legrand J. Investigation of the combined effects of acetate and photobioreactor illuminated fraction in the induction of anoxia for hydrogen production by Chlamydomonas reinhardtii. Int J Hydrogen Energy. 2010;35:10741-9.

27. Kosourov SN, Batyrova KA, Petushkova EP, Tsygankov AA, Ghirardi ML, Seibert M. Maximizing the hydrogen photoproduction yields in Chlamydomonas reinhardtii cultures: the effect of the $\mathrm{H}_{2}$ partial pressure. Int J Hydrogen Energy. 2012;37:8850-8.

28. Pringsheim E, Wiessner W. Photo-assimilation of acetate by green organisms. Nature. 1960;188:919-21.

29. Fouchard S, Hemschemeier A, Caruana A, Pruvost J, Legrand J, Happe T, et al. Autotrophic and mixotrophic hydrogen photoproduction in sulfur-deprived Chlamydomonas cells. Appl Environ Microbiol. 2005:71:6199-205.

30. Bora PL, Singh AK. New insights into designing metallacarborane based room temperature hydrogen storage media. J Chem Phys. 2013;139:164319.
31. Batyrova KA, Tsygankov AA, Kosourov SN. Sustained hydrogen photoproduction by phosphorus-deprived Chlamydomonas reinhardtii cultures. Int J Hydrogen Energy. 2012;37:8834-9.

32. Philipps $G$, Happe T, Hemschemeier A. Nitrogen deprivation results in photosynthetic hydrogen production in Chlamydomonas reinhardtii. Planta. 2012;235:729-45.

33. Volgusheva A, Kukarskikh G, Krendeleva T, Rubina A, Mamedov F. Hydrogen photoproduction in green algae Chlamydomonas reinhardtii under magnesium deprivation. RSC Advances. 2014;5:5633-7.

34. Antal TK, Krendeleva TE, Laurinavichene TV, Makarova WV, Ghirardi ML, Rubin $\mathrm{AB}$, et al. The dependence of algal $\mathrm{H}_{2}$ production on Photosystem $\mathrm{II}$ and $\mathrm{O}_{2}$ consumption activities in sulfur-deprived Chlamydomonas reinhardtii cells. Biochim Biophys Acta. 2003;1607:153-60.

35. Kosourov S, Patrusheva E, Ghirardi ML, Seibert M, Tsygankov A. A comparison of hydrogen photoproduction by sulfur-deprived Chlamydomonas reinhardtii under different growth conditions. J Biotechnol. 2007;128:776-87.

36. Laurinavichene TV, Tolstygina IV, Galiulina RR, Ghirardi ML, Seibert M, Tsygankov AA. Dilution methods to deprive Chlamydomonas reinhardtii cultures of sulfur for subsequent hydrogen photoproduction. Int J Hydrogen Energy. 2002;27:1245-9.

37. Fedorov AS, Kosourov S, Ghirardi ML, Seibert M. Continuous hydrogen photoproduction by Chlamydomonas reinhardtii: using a novel twostage, sulfate-limited chemostat system. Appl Biochem Biotechnol. 2005;121-124:403-12

38. Oncel S, Vardar-Sukan F. Photo-bioproduction of hydrogen by Chlamydomonas reinhardtii using a semi-continuous process regime. Int J Hydrogen Energy. 2009;34:7592-602.

39. Kosourov S, Makarova V, Fedorov AS, Tsygankov A, Seibert M, Ghirardi ML. The effect of sulfur re-addition on $\mathrm{H}(2)$ photoproduction by sulfurdeprived green algae. Photosynth Res. 2005;85:295-305.

40. Kosourov S, Tsygankov A, Seibert M, Ghirardi ML. Sustained hydrogen photoproduction by Chlamydomonas reinhardtii: effects of culture parameters. Biotechnol Bioeng. 2002;78:731-40.

41. Degrenne B, Pruvost J, Legrand J. Effect of prolonged hypoxia in autotrophic conditions in the hydrogen production by the green microalga Chlamydomonas reinhardtii in photobioreactor. Bioresour Technol. 2011;102:1035-43.

42. Tsygankov AA, Kosourov SN, Tolstygina IV, Ghirardi ML, Seibert M. Hydrogen production by sulfur-deprived Chlamydomonas reinhardtii under photoautotrophic conditions. Int J Hydrogen Energy. 2006;31:1574-84.

43. Tsygankov A, Kosourova S, Seibert M, Ghirardi ML. Hydrogen photoproduction under continuous illumination by sulfur-deprived, synchronous Chlamydomonas reinhardtii cultures. Int J Hydrogen Energy. 2002;27:1239-44

44. Asada Y, Miyake J. Photobiological hydrogen production. J Biosci Bioeng 1999;88:1-6.

45. Heifetz PB, Forster B, Osmond CB, Giles LJ, Boynton JE. Effects of acetate on facultative autotrophy in Chlamydomonas reinhardtii assessed by photosynthetic measurements and stable isotope analyses. Plant Physiol. 2000;122:1439-45.

46. Endo T, Asada K. Dark induction of the non-photochemical quenching of chlorophyll fluorescence by acetate in Chlamydomonas reinhardtii. Plant Cell Physiol. 1996;37:551-5.

47. Gérin S, Mathy G, Franck F. Modeling the dependence of respiration and photosynthesis upon light, acetate, carbon dioxide, nitrate and ammonium in Chlamydomonas reinhardtii using design of experiments and multiple regression. BMC Syst Biol 2014; 8:96. http://www.biomedcentral. com/1752-0509/8/96

48. Antal TK, Matorin DN, Ilyash LV, Volgusheva AA, Osipov V, Konyuhov IV, et al. Probing of photosynthetic reactions in four phytoplanktonic algae with a PEA fluorometer. Photosynth Res. 2009;102:67-76.

49. Healey FP. Hydrogen evolution by several algae. Planta. 1970;91:220-6.

50. Maione TE, Gibbs M. Hydrogenase-mediated activities in isolated chloroplasts of Chlamydomonas reinhardii. Plant Physiol. 1986;80:360-3.

51. Willeford KO, Gibbs M. Localization of the enzymes involved in the photoevolution of $\mathrm{H}(2)$ from acetate in Chlamydomonas reinhardtii. Plant Physiol. 1989;90:788-91.

52. Willeford KO, Gombos Z, Gibbs M. Evidence for chloroplastic succinate dehydrogenase participating in the chloroplastic respiratory and photosynthetic electron transport chains of Chlamydomonas reinhardtii. Plant Physiol. 1989;90:1084-7. 
53. Noth J, Krawietz D, Hemschemeier A, Happe T. Pyruvate:ferredoxin oxidoreductase is coupled to light-independent hydrogen production in Chlamydomonas reinhardtii. J Biol Chem. 2013;288:4368-77.

54. Atteia A, Adrait A, Brugiere S, Tardif M, van Lis R, Deusch O, et al. A Proteomic Survey of Chlamydomonas reinhardtii mitochondria sheds new light on the metabolic plasticity of the organelle and on the nature of the alpha-proteobacterial mitochondrial ancestor. Mol Bio Evol. 2009;26:1533-48.

55. Rolland N, Atteia A, Decottignies P, Garin J, Hippler M, Kreimer G, et al. Chlamydomonas proteomics. Curr Opin Microbiol. 2009;12:285-91.
56. Johnson X, Alric J. Central carbon metabolism and electron transport in Chlamydomonas reinhardtii: metabolic constraints for carbon partitioning between oil and starch. Eukaryot Cell. 2013;12:776-93.

57. Loppes R, Radoux M, Ohresser MC, Matagne RF. Transcriptional regulation of the Nial gene encoding nitrate reductase in Chlamydomonas reinhardtii: effects of various environmental factors on the expression of a reporter gene under the control of the Nia1 promoter. Plant Mol Biol. 1999:41:701-11.

58. Harris EH. The Chlamydomonas sourcebook. A comprehensive guide to biology and laboratory use. San Diego: Academic Press; 1989.

Submit your next manuscript to BioMed Central and take full advantage of:

- Convenient online submission

- Thorough peer review

- No space constraints or color figure charges

- Immediate publication on acceptance

- Inclusion in PubMed, CAS, Scopus and Google Scholar

- Research which is freely available for redistribution

Submit your manuscript at

www.biomedcentral.com/submit

( ) Biomed Central 